\title{
Comment on "Concepts against mathematics: self-inconsistency in thermodynamic evaluations, V. A. Drebushchak, Journal of Thermal Analysis and Calorimetry (2011) 103:753-759"
}

\author{
I. A. Stepanov
}

Received: 3 December 2013/ Accepted: 17 January 2014/Published online: 6 February 2014

(c) Akadémiai Kiadó, Budapest, Hungary 2014

In 2011, Drebushchak published his paper [1]. Swendsen already published a Comment on it [2]. However, his Comment has a disadvantage: he commented on the main body of Drebushchak's paper but did not comment on the paradox that Drebushchak had proposed in the Appendix of his paper.

I would like to Comment on this paradox (I insert the letter "D" into Drebushchak's equation numbers).

In Appendix of [1], a paradox was presented. It goes as follows. A conventional result for the ideal gas was taken: $C_{\mathrm{P}}=C_{\mathrm{V}}+R$

where $R$ is the gas constant. Both sides of this equation were multiplied by $\mathrm{d} T$ :

$C_{\mathrm{P}} \mathrm{d} T=C_{\mathrm{V}} \mathrm{d} T+R \mathrm{~d} T$,

and taking into account that $R \mathrm{~d} T=\mathrm{d} R T=\mathrm{d}(P V)$, Eq. D39 was transformed into

$C_{\mathrm{P}} \mathrm{d} T=C_{\mathrm{V}} \mathrm{d} T+\mathrm{d}(P V)$.

Then, if one divided Eq. D42 by $\mathrm{d} T$, one obtained the paradox:

$C_{\mathrm{P}}=C_{\mathrm{V}}+V \frac{\mathrm{d} P}{\mathrm{~d} T}+P \frac{\mathrm{d} V}{\mathrm{~d} T}=C_{\mathrm{V}}+2 R$.

Now let us repeat this procedure. Multiply left - hand side and right - hand side of Eq. D43 by $\mathrm{d} T$ :

$C_{\mathrm{P}} \mathrm{d} T=C_{\mathrm{V}} \mathrm{d} T+2 R \mathrm{~d} T=C_{\mathrm{V}} \mathrm{d} T+2 \mathrm{~d}(P V)$.

I. A. Stepanov ( $\square)$

Institute of Mathematical Sciences and Information

Technologies, Liepaja University, Liela 14, Liepaja LV-3401, Latvia

e-mail: igstepanov@yahoo.com
Dividing Eq. 1 by $\mathrm{d} T$, one obtains:

$C_{\mathrm{P}}=C_{\mathrm{V}}+2 V \frac{\mathrm{d} P}{\mathrm{~d} T}+2 P \frac{\mathrm{d} V}{\mathrm{~d} T}=C_{\mathrm{V}}+4 R$.

If one iterates this calculation $n$ times, one obtains

$C_{\mathrm{P}}=C_{\mathrm{V}}+2^{\mathrm{n}} R$.

Now let us take the gas constant $R$, and multiplying it by $\mathrm{d} T$, one obtains

$R \mathrm{~d} T=\mathrm{d}(P V)$.

Dividing this equation by $\mathrm{d} T$, one again obtains:

$R=V \frac{\mathrm{d} P}{\mathrm{~d} T}+P \frac{\mathrm{d} V}{\mathrm{~d} T}=2 R$.

Iterating $n$ times, one obtains

$R=2^{\mathrm{n}} R$.

It is obvious that Drebushchak's paradox does not have connection to the heat capacities but results from inappropriate use of the differential equation. Its explanation is, of course, the following one. In the left part of Eq. D42, there is the value $C_{\mathrm{P}} \mathrm{d} T$. This means that we have a system with a constant pressure and want to calculate $C_{\mathrm{P}} \mathrm{d} T$ for it, which is unknown to us. In the right part of this equation, there is a value $C_{\mathrm{V}} \mathrm{d} T$ which is known to us. To obtain $C_{\mathrm{P}} \mathrm{d} T$, we have to add to $C_{\mathrm{V}} \mathrm{d} T$ a term $\mathrm{d}(P V)$ which characterizes our system with a constant pressure. Therefore, in this term, $P$ is constant.

\section{References}

1. Drebushchak VA. Concepts against mathematics: self-inconsistency in thermodynamic evaluations. J Therm Anal Calorim. 2011;103:753-9.

2. Swendsen RH. In defense of thermodynamics, comment on concepts against mathematics: self-inconsistency in thermodynamic evaluations. J Therm Anal Calorim. 2012;110(3):1547-51. 